Annales Geophysicae (2002) 20: 29-40 C) European Geophysical Society 2002

\title{
An unusual geometry of the ionospheric signature of the cusp: implications for magnetopause merging sites
}

\author{
G. Chisham $^{1}$, M. Pinnock ${ }^{1}$, I. J. Coleman ${ }^{1}$, M. R. Hairston ${ }^{2}$, and A. D. M. Walker ${ }^{3}$ \\ ${ }^{1}$ British Antarctic Survey, Natural Environment Research Council, High Cross, Madingley Road, Cambridge, CB3 0ET, UK \\ ${ }^{2}$ Center for Space Sciences, The University of Texas at Dallas, Richardson, Texas, USA \\ ${ }^{3}$ School of Pure and Applied Physics, University of Natal, Durban 4041, South Africa
}

Received: 24 January 2001 - Revised: 11 July 2001 - Accepted: 17 September 2001

\begin{abstract}
The HF radar Doppler spectral width boundary (SWB) in the cusp represents a very good proxy for the equatorward edge of cusp ion precipitation in the dayside ionosphere. For intervals where the Interplanetary Magnetic Field (IMF) has a southward component $\left(B_{z}<0\right)$, the SWB is typically displaced poleward of the actual location of the open-closed field line boundary (or polar cap boundary, PCB). This is due to the poleward motion of newlyreconnected magnetic field lines during the cusp ion travel time from the reconnection $\mathrm{X}$-line to the ionosphere. This paper presents observations of the dayside ionosphere from $\mathrm{Su}-$ perDARN HF radars in Antarctica during an extended interval $(\sim 12 \mathrm{~h})$ of quasi-steady IMF conditions $\left(B_{y} \sim B_{z}<0\right)$. The observations show a quasi-stationary feature in the SWB in the morning sector close to magnetic local noon which takes the form of a $2^{\circ}$ poleward distortion of the boundary. We suggest that two separate reconnection sites exist on the magnetopause at this time, as predicted by the anti-parallel merging hypothesis for these IMF conditions. The observed cusp geometry is a consequence of different ion travel times from the reconnection $\mathrm{X}$-lines to the southern ionosphere on either side of magnetic local noon. These observations provide strong evidence to support the anti-parallel merging hypothesis. This work also shows that mesoscale and smallscale structure in the SWB cannot always be interpreted as reflecting structure in the dayside PCB. Localised variations in the convection flow across the merging gap, or in the ion travel time from the reconnection X-line to the ionosphere, can lead to localised variations in the offset of the SWB from the PCB. These caveats should also be considered when working with other proxies for the dayside PCB which are associated with cusp particle precipitation, such as the 630 $\mathrm{nm}$ cusp auroral emission.
\end{abstract}

Key words. Ionosphere (plasma convection) - Magnetospheric physics (magnetopause, cusp, and boundary layers)

Correspondence to: G. Chisham (G.Chisham@bas.ac.uk)
- Space plasma physics (magnetic reconnection)

\section{Introduction}

Magnetic reconnection (merging) at the magnetopause is the dominant means by which energy is transferred from the solar wind into the Earth's magnetosphere and is the major driver of the global magnetospheric convection process. On the dayside magnetopause, reconnection between magnetosheath, and closed magnetospheric magnetic field lines occurs preferentially when the interplanetary magnetic field (IMF) has a southward component $\left(B_{z}<0\right)$. Newlyreconnected magnetic field lines map to the cusp regions of the dayside ionosphere (Smith and Lockwood, 1996 present a recent review of cusp-related science). Magnetosheathlike plasma travels down these field lines, precipitating in the ionosphere and resulting in the cusp aurora. The newlyreconnected field lines are swept across the polar regions of the magnetosphere to the nightside of the Earth by the magnetosheath flow. Following further reconnection in the magnetospheric tail, they return to the dayside magnetosphere, completing a global convection system which maps to twocell convection patterns in the polar ionospheres.

The global convection process and its ionospheric signature vary significantly with IMF magnitude and direction (Crooker, 1979; Heelis, 1984; Reiff and Burch, 1985). The convection streamlines in the ionosphere describe equipotential contours of the convection electric field and the potential between the centres of the two convection cells (termed the cross-polar cap potential) describes the intensity of the global convection system. Empirical models of the ionospheric electric equipotential pattern, parameterized by IMF varaitions (Ruohoniemi and Greenwald, 1996; Weimer, 2001), illustrate clearly the spatial variation of this two-cell convection pattern for different IMF magnitude and direction. The cross-polar cap potential also varies considerably in 


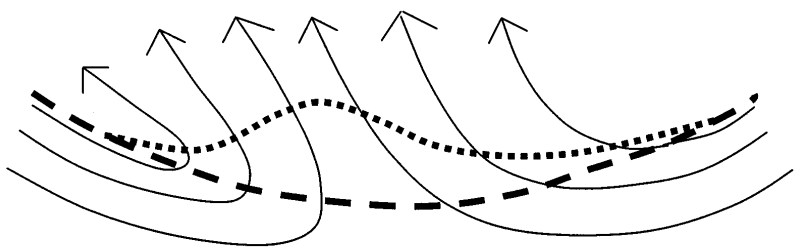

\section{- - Polar Cap Boundary ..... Proxy (Spectral Width Boundary)}

Fig. 1. A schematic representation of the relationship between the spectral width boundary and the actual polar cap boundary in the ionospheric cusp region. The spectral width boundary is offset from the polar cap boundary in regions where the convection flow has a poleward component.

magnitude, and correlates well with the interplanetary electric field input from the solar wind (Reiff et al., 1981; Doyle and Burke, 1983; Wygant et al., 1983). These studies have suggested a functional form for the cross-polar cap potential which has a linear dependence on the interplanetary electric field (Burke et al., 1999). The interplanetary electric field depends on IMF $B_{y}$ and $B_{z}$, and the solar wind velocity (Kan and Lee, 1979). As a general rule, as IMF $B_{z}$ becomes more negative, the polar cap potential increases.

The footprint of the open-closed field line boundary in the ionosphere is termed the polar cap boundary (PCB). The continual addition and removal of flux across this boundary, as a result of reconnection, is described in the expanding/contracting polar cap model (Siscoe and Huang, 1985; Cowley and Lockwood, 1992). The reconnection location (termed the $\mathrm{X}$-line) on the magnetopause maps to a region in the dayside ionosphere termed the merging gap (Moses et al., 1987) which is a longitudinally-limited region of the PCB through which the ionospheric plasma flows into the polar cap. Identifying the PCB in the dayside ionosphere is crucial for studying the ionospheric signature of magnetic reconnection on the dayside magnetopause. At present, it is not always possible to directly observe the PCB in the dayside ionosphere, although there are features that provide proxies for the PCB position in the cusp (Rodger, 2000):

1. The equatorward boundary of the $630 \mathrm{~nm}$ cusp aurora (Milan et al., 1999);

2. The equatorward edge of the high spectral width region in HF radar data (termed the spectral width boundary, SWB) (Baker et al., 1995; Rodger et al., 1995), and

3. The equatorward edge of cusp ion precipitation in low altitude satellite observations (Newell and Meng, 1991).

These features represent observations of the equatorward boundary of the cusp particle precipitation and are hence displaced poleward from the true PCB due to time-of-flight effects of the precipitating particles. The displacement depends on a number of factors:
1. The field-aligned distance from the reconnection $\mathrm{X}$-line on the magnetopause to the ionosphere (which determines the cusp ion travel time);

2. The reconnection electric field, which determines the speed of the flow through the merging gap;

3. The energy and pitch-angle distributions of the precipitating cusp ions which will also influence the ion travel time along the field line.

Recent work (Baker et al., 1997; Provan et al., 1998; Pinnock et al., 1999; Chisham et al., 2001) has shown the importance of using the SWB as a proxy for the PCB in the cusp. There is a growing understanding of the factors which result in the high spectral width values observed in the cusp region (André et al., 1999, 2000) and also those which influence the offset of the SWB from the actual PCB (Rodger and Pinnock, 1997; Lockwood, 1997; Rodger, 2000; Pinnock and Rodger, 2001). However, little attention has been paid to the effect of temporal and spatial variations in the factors influencing the offset. Understanding the factors that influence the offset is important if one wants to interpret small or mesoscale variations in the SWB, i.e. to distinguish genuine variations in the PCB from variations in the offset between the PCB and its proxy. Figure 1 displays a simplified schematic illustration of the relationship between the PCB and the SWB (or other proxy) in the cusp region (Rodger, 2000). The SWB is displaced from the PCB only within the ionospheric footprint of the merging gap where there is a poleward flow component across the PCB. Hence, the SWB variation can potentially be used as a diagnostic of the extent of the merging gap by identifying the longitudinal extent of the poleward displacement. In practice, this is rarely feasible, as the merging gap is typically much larger than the field of view of a single instrument; the extent of the merging gap is thought to be typically $\sim 3 \mathrm{~h}$ of magnetic local time (MLT) (Lockwood and Davis, 1996), or longer (Maynard et al., 1997; Nishitani et al., 1999). Pinnock and Rodger (2001) have observed this characteristic proxy variation in the combined SWB variation of four SuperDARN HF radars covering $\sim 6 \mathrm{~h}$ of MLT.

In this paper, we study an extended interval of extremely steady solar wind and IMF conditions $\left(B_{z} \sim B_{y} \sim-10 \mathrm{nT}\right)$ during which the Halley HF radar in Antarctica observed a very localised $2^{\circ}$ poleward distortion of the SWB in the morning sector close to magnetic local noon. The distortion was quasi-stationary in magnetic local time with a width of $\sim 1 \mathrm{~h}$ of MLT, and lasted for at least an hour (as the Halley radar field of view passed through it). By invoking the antiparallel merging hypothesis (Crooker, 1979), we show that this distortion can be explained as a result of a localised increase in the displacement of the SWB from the PCB in the morning sector and does not represent structure in the PCB. This increased displacement results from the morning sector reconnection X-line on the magnetopause being located further from the Southern Hemisphere ionosphere than its afternoon sector counterpart, as predicted by the anti-parallel merging hypothesis during intervals when IMF $B_{z} \sim B_{y}$. 


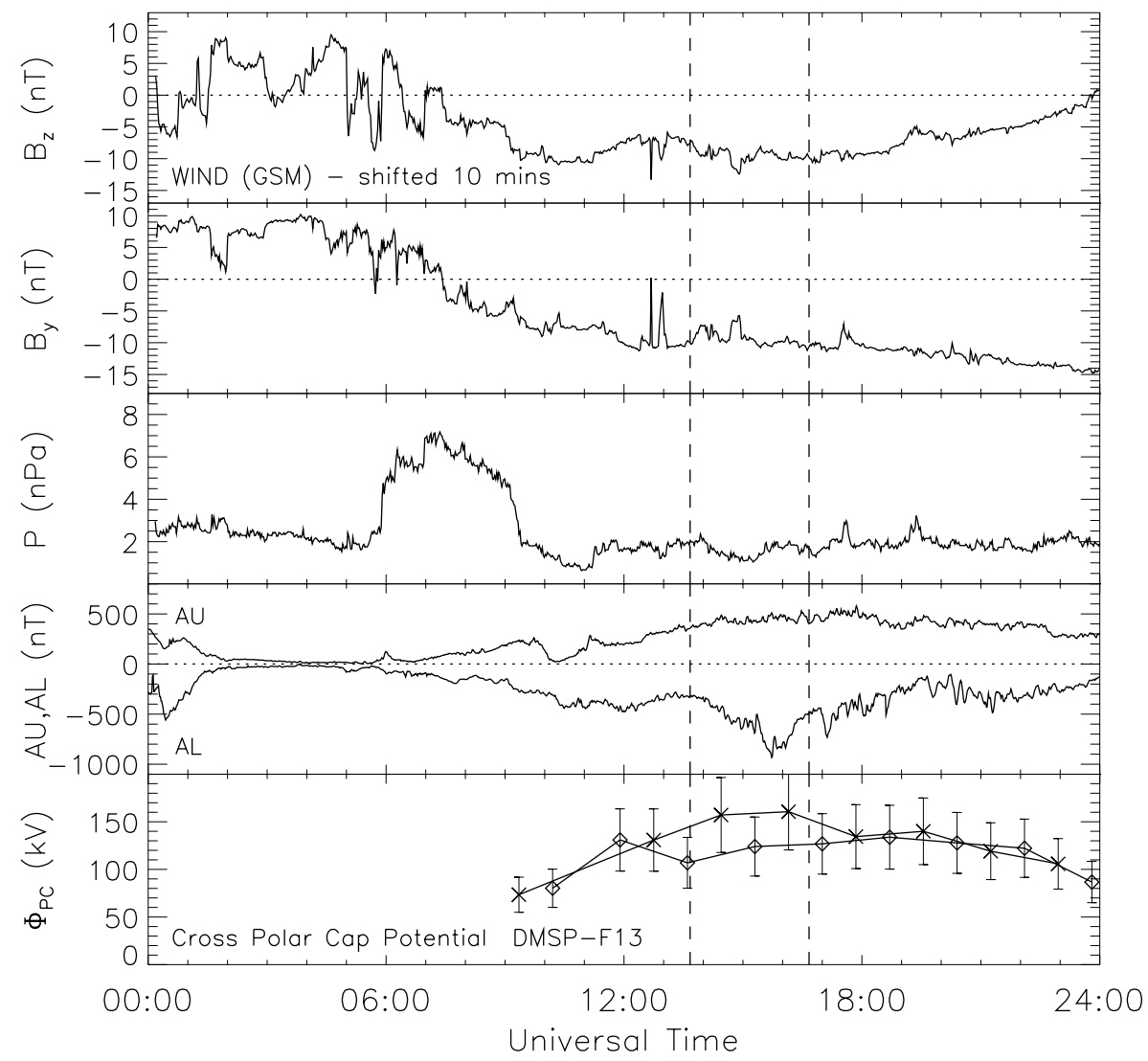

Fig. 2. Data presenting an overview of solar wind and magnetospheric variations on 20 August 1998. The panels show the interplanetary magnetic field $B_{z}$ and $B_{y}$ components (in GSM coordinates) and solar wind dynamic pressure from the WIND spacecraft, the $\mathrm{AU}$ and $\mathrm{AL}$ index variations, and the cross-polar cap potential estimate from DMSP-F13.
This results in a longer cusp ion travel time and hence, a greater displacement between the PCB and the SWB. By showing that the observed mesoscale variations in the SWB do not imply variations in the PCB on this occasion, we also demonstrate the importance of understanding the offset between the PCB and its proxies.

\section{Instrumentation}

This study presents data from the SHARE (Southern Hemisphere Auroral Radar Experiment) HF radar pair located in Antarctica. The SHARE Halley and Sanae radars are part of the SuperDARN radar network. SuperDARN (The Super Dual Auroral Radar Network) is a network of coherent scatter HF radars (Greenwald et al., 1995) which measure backscatter from magnetic field-aligned decametrescale ionospheric irregularities. The radars transmit HF signals which are refracted towards the horizontal as they enter ionospheric regions with higher electron concentrations. If these regions contain irregularities, the radar signals are backscattered when they are propagating perpendicular to the magnetic field (i.e. perpendicular to the irregularities). In the high-latitude ionosphere, these irregularities are often present (Tsunoda, 1988); they move with the background plasma drift at F-region altitudes (Villain et al., 1985; Ruohoniemi et al., 1987) and hence, provide information about large-scale convection and related processes in the radar field of view. The radars transmit power at a fixed frequency in the range of $8-20 \mathrm{MHz}$ and from the return signals, an estimate of the variation in backscatter power, line-of-sight Doppler velocity and Doppler spectral width in the radar field of view is derived (Baker et al., 1995 for details). Many of the SuperDARN radars have overlapping fields of view which means that line-of-sight Doppler velocities from these radars can be merged to produce two-dimensional velocity vectors.

The SHARE Halley radar is located at Halley, Antarctica $\left(75.5^{\circ} \mathrm{S},-26.6^{\circ} \mathrm{E}\right)$, and transmits in the direction of the geomagnetic South Pole. The radar scan sweeps through 16 beam positions differing by $3.25^{\circ}$ in azimuth. On the day studied in this paper (20 August 1998), the radar was operating in a special mode which included three beams running at a high time resolution. The integration time was $5 \mathrm{~s}$ for each beam and a full scan was made in $3 \mathrm{~min}$, with each of the high time resolution beams repeating every $30 \mathrm{~s}$. Each beam comprised 75 range gates, with a pulse length of $300 \mu \mathrm{s}$ (equivalent to $45 \mathrm{~km})$ and a lag to the first range of $1200 \mu \mathrm{s}(180$ $\mathrm{km})$. The SHARE Sanae radar $\left(72.0^{\circ} \mathrm{S},-3.0^{\circ} \mathrm{E}\right)$ shares a large, common field of view with the Halley radar and was operating in a similar mode at this time. Line-of-sight velocities from the Sanae radar were merged with those from Halley to produce field-perpendicular two-dimensional velocity vectors in the overlap region.

The SWB represents the boundary between the high spectral width values associated with regions of cusp ion pre- 

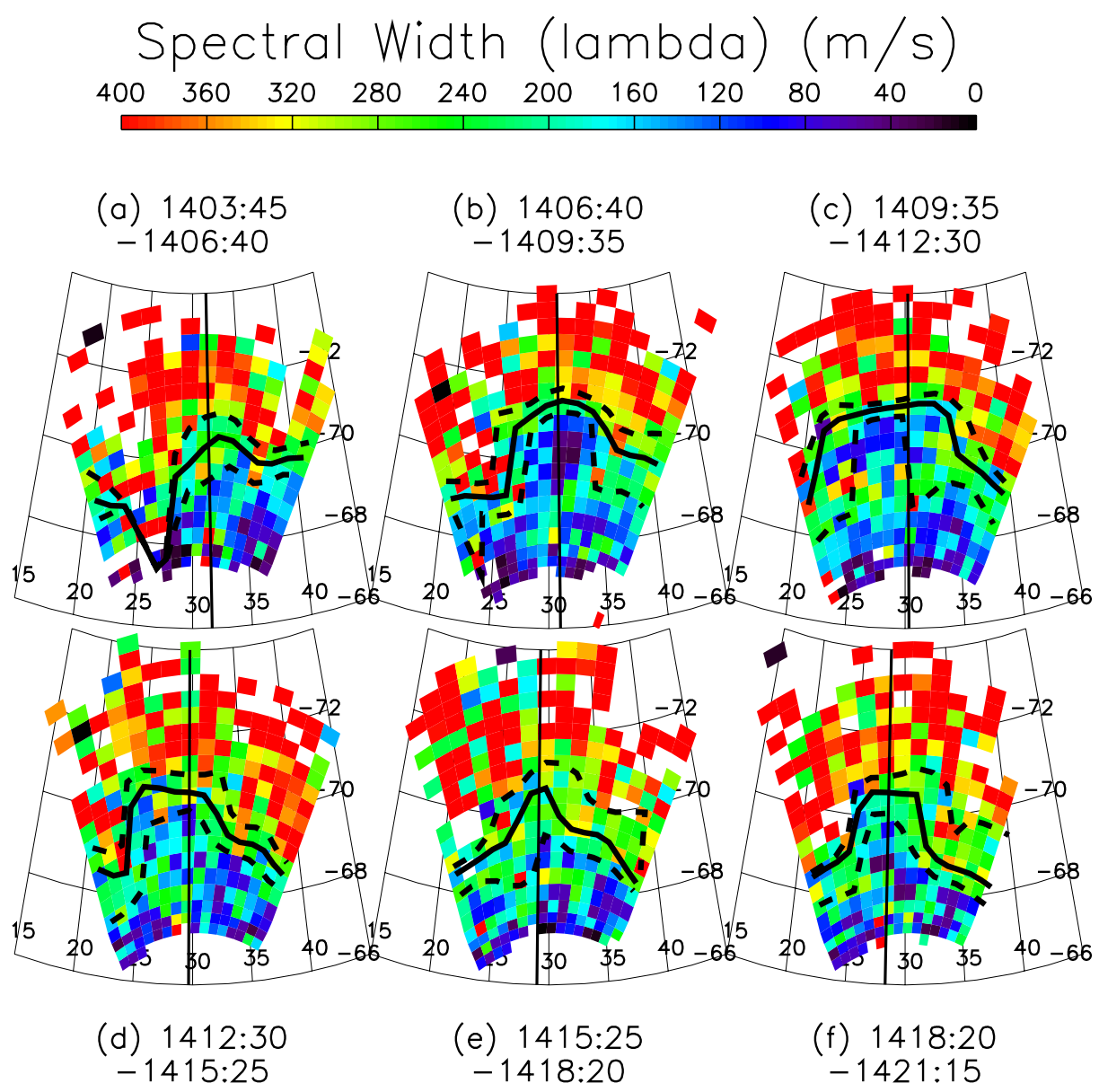

Fig. 3. The spectral width variation in six consecutive scans from the Halley radar on 20 August 1998. The bold line enclosed by two dashed lines represents the spectral width boundary and its uncertainty, respectively. The solid vertical line marks the longitude of 11:00 MLT.

cipitation and the low values observed in the regions equatorward of the cusp (Baker et al., 1995). The wide and complex Doppler spectra which characterise the cusp result from electric field variations in the Pc1-2 frequency range ( $\sim 0.1-5 \mathrm{~Hz})$ (André et al., 1999) which are thought to be related to the cusp precipitation. The spectral width is highly variable throughout the cusp; its distribution is approximately Gaussian in nature, ranging from $\sim 100-600 \mathrm{~m} / \mathrm{s}$. Regions equatorward of the cusp are typically characterised by narrow Doppler spectra dominated by a single component. The spectral width in these regions is generally very low $(<100 \mathrm{~m} / \mathrm{s})$. Generally, the SWB is clearly defined at the equatorward edge of the ionospheric cusp. However, it must be stressed that caution is needed when interpreting high spectral widths in HF radar data, since there are a number of other sources of high spectral width unrelated to the cusp (André et al., 2000).

In this paper, we determine the SWB in the radar data using the method outlined by Chisham et al. (2001). In this method, the spectral width variation is first spatially smoothed and the SWB in each beam is taken as the point at which the smoothed spectral width variation first crosses $250 \mathrm{~m} / \mathrm{s}$. The method also requires that the average spec- tral width over three range gates poleward of the suspected boundary position is $>250 \mathrm{~m} / \mathrm{s}$ to eliminate the effect of occasional spectral width peaks at low ranges.

\section{Event overview}

On 20 August 1998, the WIND spacecraft was monitoring the solar wind approximately $65 R_{E}$ upstream of the Earth, displaced from the Earth-Sun line by $\sim 35 R_{E}$ in the negative GSE $y$-direction. Figure 2 presents IMF $B_{z}$ and $B_{y}$ and the solar wind dynamic pressure variations observed by WIND on this day, in the GSM coordinate system. The GEOTAIL spacecraft, located approximately $30 R_{E}$ upstream on the Earth-Sun line, observed almost identical variations in these parameters with no large offset in the timing of significant features in the early part of the day. However, a large data gap occurred in the GEOTAIL data between 12:00 and 19:30 UT and hence, the WIND data are shown. Since the variations seen by both spacecraft were so similar, we can be confident that despite its offset from the Earth-Sun line, the WIND observations describe well the solar wind that interacts with the Earth's magnetosphere on this day. The two 
spacecraft observations provide us with the means to make an accurate determination of the orientation of the solar wind phase front in the $x-y$ plane on this day, allowing for an estimate to be made of the time delay from WIND to the ionospheric observations. We assume that any variation in the phase front in the $z$-direction will not significantly affect our time delay estimate. The WIND data in Fig. 2 have been shifted by $10 \mathrm{~min}$ to allow for the estimated time delay. Our ionospheric observations were predominantly confined to 13:40-16:40 UT (demarked by the vertical dashed lines in Fig. 2), an interval when the solar wind was very steady. Any uncertainty which exists in the time delay estimation is, therefore, of little importance to this study. The constant solar wind and IMF conditions allow us to speculate that the magnetic reconnection scenario at the dayside magnetopause, and hence its ionospheric signature, remained quasi-steady during this interval.

During such an extended interval of large negative IMF $B_{z}$, the magnetosphere is usually very dynamic, characterised by a number of substorm intervals. Figure 2 also shows the AU and AL index variations on 20 August 1998. After 06:00 UT, the AE index (AU-AL) grows steadily following the southward turning of the IMF. However, there is little substorm activity; only minor substorms are evident, most notably at 15:20 UT and 17:00 UT. Much of the interval resembles an interval of "Steady Magnetospheric Convection" (SMC) (Sergeev et al., 1996 for a recent review). SMC events are defined as periods of enhanced energy input from the solar wind into the magnetosphere over a time interval of several substorm time constants during which the largescale stability of the magnetotail is sustained. For example, Yahnin et al. (1994) observed an interval of intense magnetospheric convection which continued for more than $10 \mathrm{~h}$ under steady southward IMF conditions without any distinct substorm signatures. They observed that the dayside cusp was located at an unusually low-latitude $\left(\sim 70^{\circ}\right)$ at this time. The existence of predominantly steady convection and minimal substorm activity during our event further suggests that the dayside convection scenario will be quasi-steady throughout this interval.

The final panel in Fig. 2 presents an estimate of the crosspolar cap potential variation from DMSP-F13. The DMSP spacecraft describe low altitude polar orbits of the Earth and measure plasma characteristics in the polar regions. DMSPF13 is the operational DMSP spacecraft which was closest to a dawn-dusk orientation at this time and provided an estimate of the cross-polar cap potential variation for our interval of study. Two data sets are presented; the diamonds represent northern hemisphere measurements, whereas the crosses represent southern hemisphere measurements. The values shown represent the observed potential variation along the spacecraft track, which is always less than the true total potential (Hairston et al., 1998). Typically, if the spacecraft orbit passes above $80^{\circ}$ magnetic latitude, then the measurement will represent $80-95 \%$ of the true potential. For the data shown, only three of the measurements were taken from orbital passes that did not exceed $80^{\circ}$ and hence, the ob-

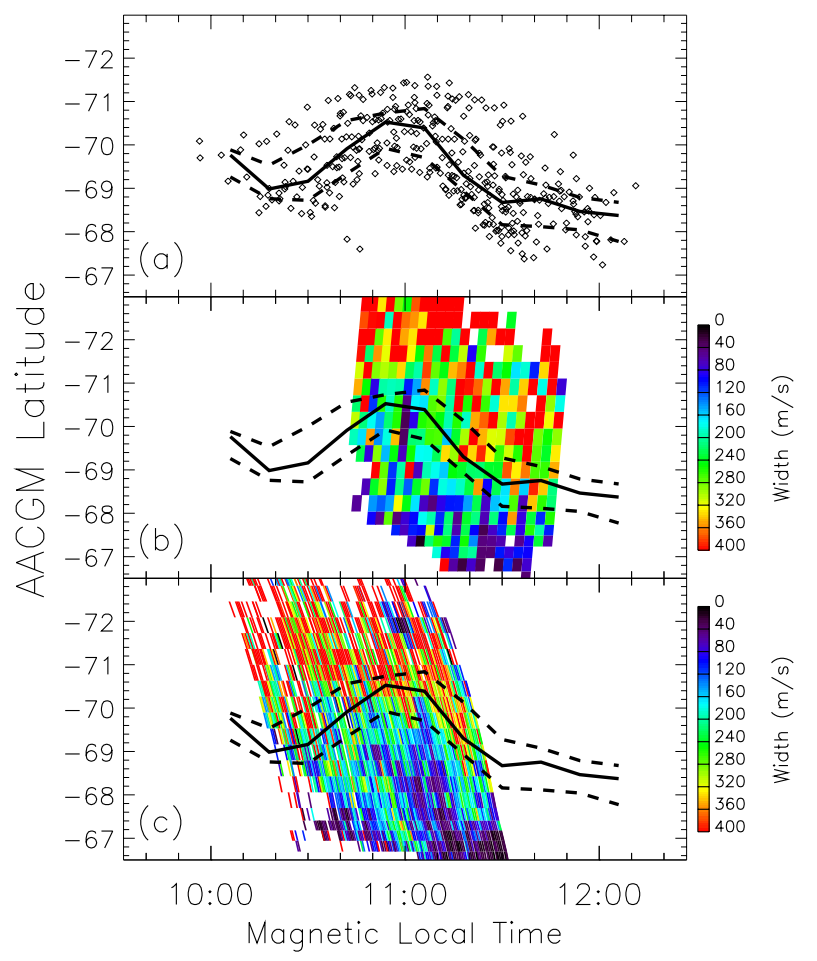

Fig. 4. (a) The statistical variation of the spectral width boundary position with magnetic local time during the interval 13:5015:00 UT. Each symbol represents a single observation of the spectral width boundary taken from a single beam from a single 3-min scan. (b) The spectral width variation seen by Halley beam 8 during the interval 13:50-15:00 UT. (c) The spectral width variation seen by beam 3 during the interval 13:50-15:00 UT. The solid and dashed lines on all the panels represent the median and quartile variations of the scatterplot observations.

served variation represents a true reflection of the cross-polar cap potential on this day. The potential measured is very large, typically $\sim 100-150 \mathrm{kV}$, which is not unusual during extended intervals of IMF $B_{z} \ll 0$. There is very little variation in the potential during the steady solar wind interval; the potential is smaller at the start of the interval of data shown (immediately after the $B_{z}$ southward turning) and at the end of the interval of data (as $B_{z}$ approaches 0). During the interval when we observed our strong radar backscatter (13:4016:40 UT), the observed potential was $\sim 100-160 \mathrm{kV}$. The difference between the observed potentials in the northern and southern hemispheres during this interval is attributed more to the differences in the satellite's track across the polar cap regions in the two hemispheres, rather than to any intrinsic difference in the total potentials in each hemisphere.

\section{Ionospheric observations}

\subsection{Spectral width boundary observations}

On this day, the Halley and Sanae HF radars observed almost $3 \mathrm{~h}(\sim 13: 40$ to $16: 40 \mathrm{UT})$ of continuous backscatter 


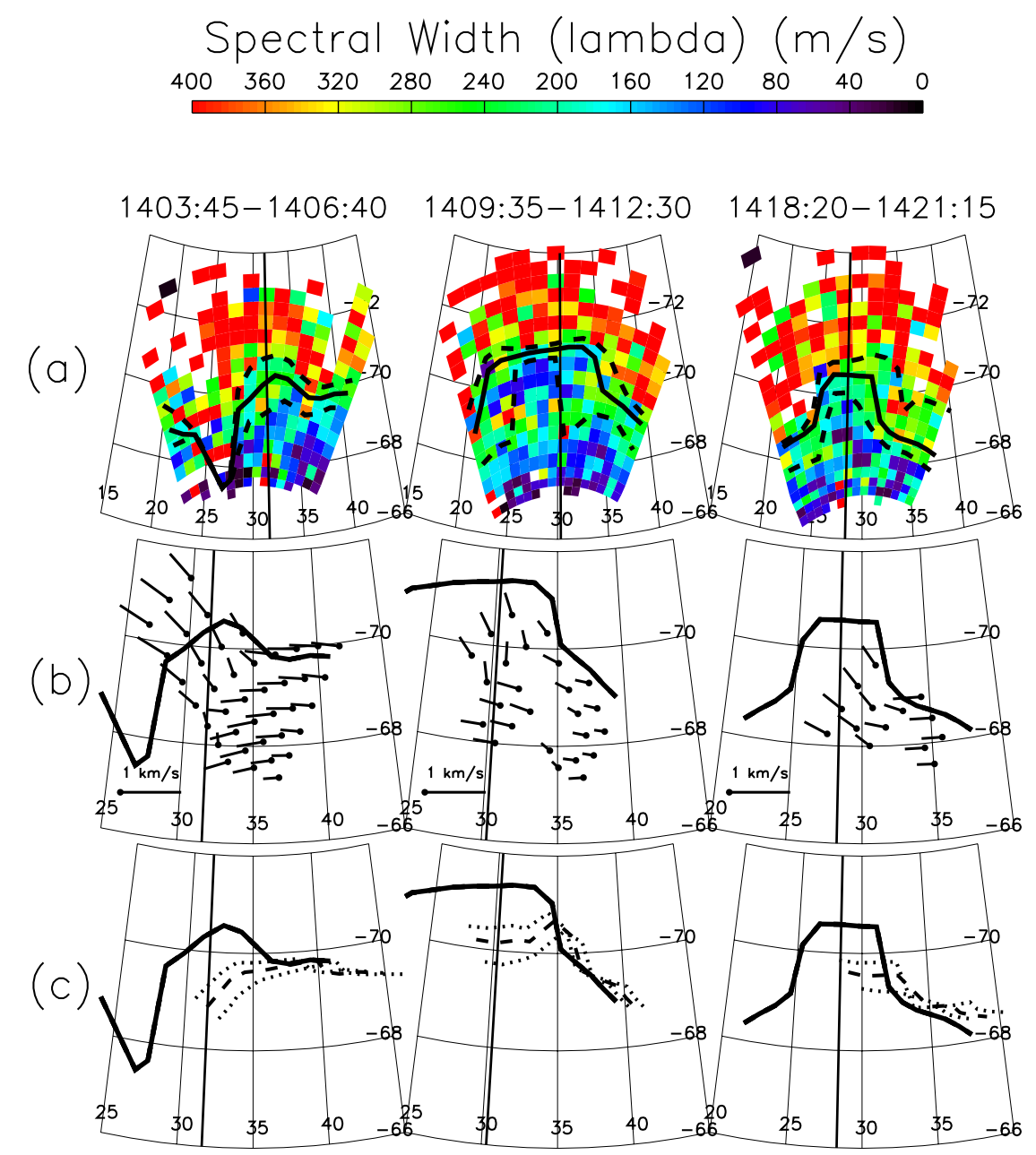

Fig. 5. Data from three Halley radar scans during the interval 13:50-15:00 UT (times shown). (a) The spectral width variation. The bold and dashed lines represent the spectral width boundary (see text). (b) Comparison of merged velocity vectors and the spectral width boundary. (c) Comparison of the spectral width boundary (bold line) with the mapped estimates of the polar cap boundary (dashed and dotted lines, see text).

close to magnetic local noon (magnetic local noon at Halley was at $\sim 15: 20$ UT). During the interval 13:50-15:00 UT, the SWB variations in the 3-min full scans from the Halley and Sanae radars were characterised by $\mathrm{a} \sim 2^{\circ}$ poleward distortion of the boundary. This distortion was approximately stationary in MLT, being $\sim 1 \mathrm{~h}$ wide and centred at about 11:00 MLT. Figure 3 presents six consecutive scans from the Halley radar during the centre of this interval illustrating the spectral width variation across the field of view. The bold line surrounded by two dashed lines represents the location of the SWB and its uncertainty (as described by Chisham et al., 2001). This boundary provides a clear demarcation line between the high spectral width values in the cusp (predominantly red) and the lower spectral width values equatorward of the cusp (predominantly blue). The solid vertical line on each scan represents the longitude of 11:00 MLT. Each scan clearly shows the poleward distortion of the boundary centred on 11:00 MLT, although it does show the existence of small, temporal variations in its morphology. This fea- ture was continually observed across the interval of 13:5015:00 UT, by the Halley radar, as its field of view passed over it. The afternoon edge of the feature was also continually observed by the Sanae radar.

To illustrate the stability of this SWB feature, Fig. 4a presents a scatter plot of all the SWB positions estimated during this interval (for all 16 beams of the Halley radar in all the 3-min scans between 13:50 and 15:00 UT). The solid and dashed lines represent the median as well as the upper and lower quartile variations of the boundary position with magnetic local time. It is clear from the figure that the poleward distortion of the boundary is a stationary feature during this interval. The scatter of the boundary positions results from the small, temporal variations in the boundary distortion, as shown in Fig. 3. To illustrate further the stability of this feature, Figs. $4 \mathrm{~b}$ and $4 \mathrm{c}$ show the spectral width variations for beams 8 (at 3-min resolution) and 3 (at 30-s resolution) of the Halley radar for the same time interval. The median and quartile variations from the scatter plot have been overplot- 
ted on the spectral width data. The same variation is clearly visible in the measurements from these beams.

\subsection{Convection flow observations}

We now study the convection flow in the vicinity of the boundary distortion. Figure 5 presents the details of three of the individual 3-min scans from the Halley radar during this interval. Figure 5a presents the spectral width variations for these scans, as shown already in Fig. 3. Figure 5b compares enlarged sections of the measured boundaries (bold lines) with two-dimensional velocity vectors estimated by merging the line-of-sight Doppler velocities from the Halley and Sanae radars. A clear pattern in the velocity field is apparent for all the scans during this interval where merged vectors could be estimated, including the three examples shown. Equatorward of the distortion of the SWB, the vectors are almost exclusively westward. In the locality of the boundary distortion, the vectors possess a significant poleward component and the flow across the SWB appears predominantly confined to this region. This scenario is suggestive of the schematic illustration in Fig. 1 and could imply that the distortion represents the extent of the merging gap on this day. This is considered in Sect. 4.4 of the paper. No merged vectors could be estimated outside the vicinity of this feature due to the lack of overlapping backscatter in the Halley and Sanae fields of view at earlier and later universal times. Hence, the full extent of the merging region in the dayside ionosphere could not be estimated from the radar data.

As discussed by André et al. (2000), spatial gradients in the large-scale convection pattern can enhance the spectral width values measured by the SuperDARN radars. Large gradients in the flow direction or magnitude within a single range cell can introduce several components into the spectrum measured in that range cell. Since the analysis method that is used to derive the spectral width is not well adapted to these spectra, the resulting spectral width will be enhanced. André et al. (2000) showed that a zonally-looking radar might measure a spectral width of $\sim 250 \mathrm{~m} / \mathrm{s}$ in the region of a convection reversal or large shear in the velocity field. However, for a poleward-looking radar (such as the Halley radar), the spectral width obtained from the same region is reduced (to $\sim 150 \mathrm{~m} / \mathrm{s}$ ) due to the change in the geometry of the range cell with respect to the velocity shear. André et al. (2000) concluded that for meridionally pointing radars, the large spectral widths measured in the cusp could not be explained by gradients in the large-scale convection pattern. The effect of spatial variations in the convection flow on the spectral width can be seen in Fig. 5. All three scans show a clear change in the convection flow from westward to poleward in Fig. 5b, as discussed above. At these points there is a clear enhancement in the spectral width in Fig. 5a to $\sim 150-250 \mathrm{~m} / \mathrm{s}$ (green shading). However, gradients in the convection flow cannot be used to explain the much larger spectral width values $(>400 \mathrm{~m} / \mathrm{s})$ observed above the estimated SWB, which are a result of Pc1/2 wave activity associated with cusp precipitation.

\subsection{Estimating the polar cap boundary position}

Using the merged velocity vector measurements, the SWB positions have been reverse mapped in order to determine an estimate of the PCB position (Pinnock and Rodger, 2000). To perform this mapping, an estimate of the magnetosheathlike ion travel time from the reconnection $\mathrm{X}$-line to the ionosphere is required. Here, we assume the anti-parallel merging hypothesis and use the Tsyganenko 96 magnetospheric field model (Tsyganenko, 1995; Tsyganenko and Stern, 1996) to determine the field-aligned distance from this ionospheric location to the estimated anti-parallel merging region on the magnetopause (Coleman et al., 2000, for full details of the determination of anti-parallel regions). For the prevailing IMF conditions, this distance was estimated to be $\sim 20.4-21.3 R_{E}$ (see discussion section for further details). DMSP-F14 cusp particle observations at this time (not shown) suggested that the maximum cusp ion energies were in the range of $0.25-1.0 \mathrm{keV}$. These parameters predict an ion travel time from the reconnection X-line to the southern ionosphere of $\sim 300-600 \mathrm{~s}$ and hence, the offset of the SWB from the actual PCB will result from the motion of the newlyreconnected field lines with the convection flow during this time.

Figure 5c presents the results of mapping the observed SWBs using a velocity field determined from the measured vectors. Only the parts of the boundaries with local merged velocity vectors were mapped. In Fig. 5c, the two dotted lines represent the mapped boundaries (PCB estimations) for $300 \mathrm{~s}$ and $600 \mathrm{~s}$ ion travel times and the dashed line for $450 \mathrm{~s}$. The second and third scans presented involve greater uncertainty in the mapped boundary position due to the necessary extrapolation of the velocity field from the most poleward vectors to the observed SWB position. It is likely that the boundary offsets have been underestimated in these cases; the lineof-sight velocity variations show increasing poleward flows with latitude. It is also unlikely that the actual PCB position would be located poleward of the SWB position, as seen at the eastward edge of the second and third scans. This is most likely a result of uncertainties in the velocity field and measured SWB locations.

Although there exist uncertainties in the mapped boundary locations, a number of features of the estimated PCB positions appear robust. First, the poleward distortion, a persistent characteristic of the observed SWB, has been largely reduced. In the vicinity of the distortion, the PCB estimate is a significant distance equatorward of the observed SWB $\left(\sim 1^{\circ}\right)$, whereas outside this region, the boundary is relatively unchanged. This has largely removed the sharp poleward variation in the boundary at the edges of the distortion. Second, the PCB estimates appear to be located close to the change in the convection flow direction from westward to poleward, which is clearly apparent in the velocity vectors displayed in Fig. 5b. 


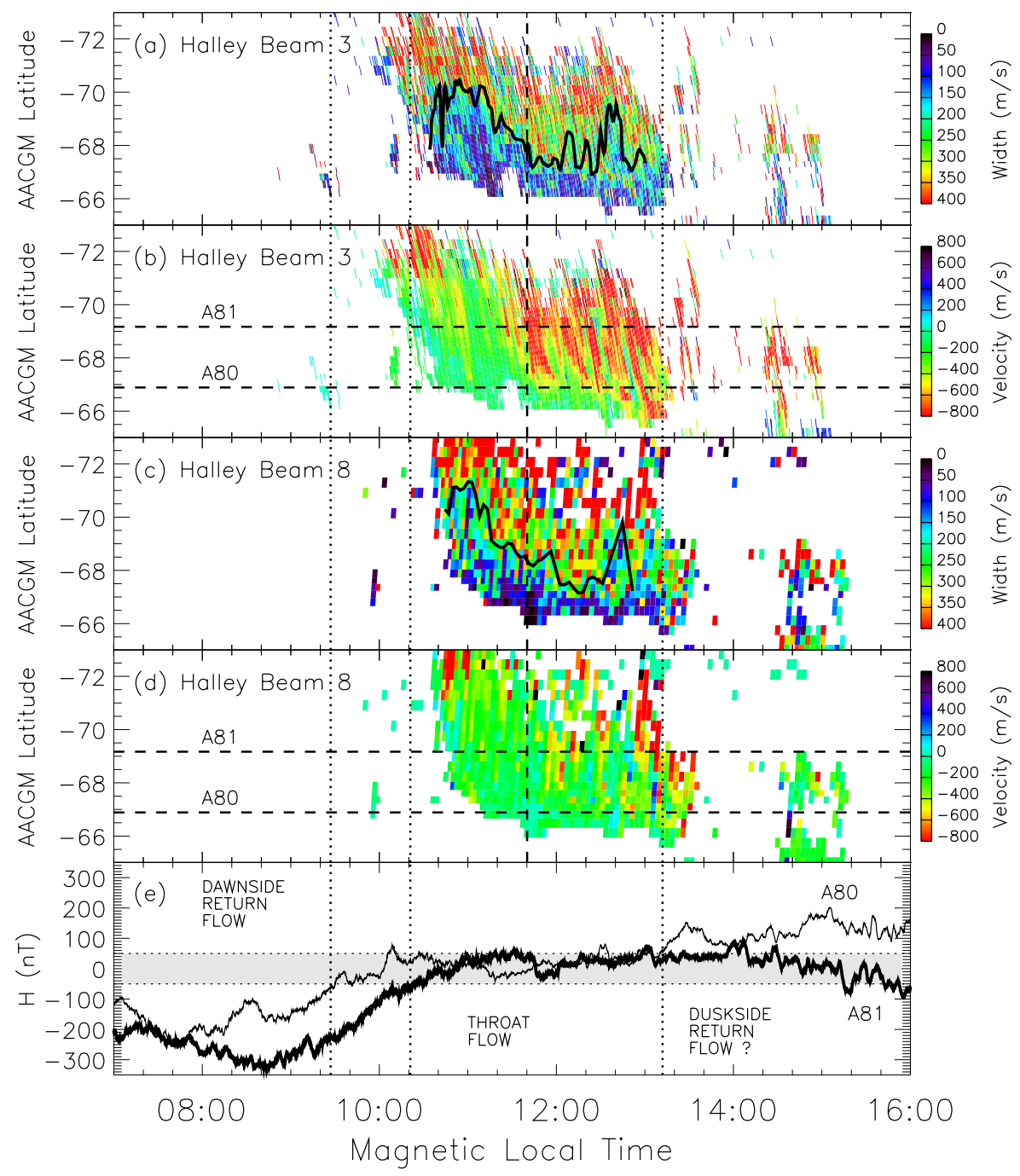

Fig. 6. (a) The spectral width variation seen by Halley beam 3 . The bold line represents an estimate of the spectral width boundary from the spectral width observations. (b) The Doppler line-of-sight velocity variation seen by Halley beam 3. The vertical dashed line represents the transition between the two flow regimes seen by beam 3 . The horizontal dashed lines denote the latitudes of the A80 and A81 magnetometers. (c) The spectral width variation seen by Halley beam 8 . The bold line represents an estimate of the spectral width boundary from the spectral width observations. (d) The Doppler line-of-sight velocity variation seen by Halley beam 8 . (e) $H$-component magnetometer data from the A80 and A81 (bold line) AGO magnetometers in the Antarctic. The vertical dotted lines delineate estimates of the boundaries between the throat and return flow as estimated from the magnetometer data.

\subsection{The morning sector reconnection potential}

We suggested earlier that poleward excursions of the SWB, such as observed here, can be used as a diagnostic of the extent of the merging gap (Rodger, 2000; Pinnock and Rodger, 2001). However, our velocity vector observations extend for less than $2 \mathrm{~h}$ of MLT and we are unable to determine the twodimensional velocity field for large regions of the dayside ionosphere. However, we can use our velocity vector measurements and our estimated PCB positions to determine the reconnection potential in our field of view (using the method employed by Pinnock et al., 1999). For all the scans during this interval for which reconnection electric field estimates could be made, the reconnection potential estimate across the field of view $(\sim 1.0-1.5 \mathrm{~h}$ of MLT) was never greater than $20 \mathrm{kV}$. Indeed, it was often less than $10 \mathrm{kV}$. The crosspolar cap potential measurements from DMSP-F13 at this time suggest a cross-polar cap potential of $\sim 100-150 \mathrm{kV}$ (see Fig. 2). This suggests that the extent of the whole merging region in the dayside ionosphere is much larger than the SWB distortion observed here. Consequently, the nature of the observed morning sector merging region suggests the existence of mesoscale variations in the overall dayside merging scenario.

\subsection{The extent of the dayside merging region}

To understand the scenario on this day, we need to obtain a large-scale picture of the dayside convection flow. So far, we have studied in detail just over an hour of data from the SHARE radar pair. Figure 6 presents the full three hours of data for this interval from Halley beam 3 (at high time resolution) and beam 8 (at scan resolution) in geomagnetic latitude and magnetic local time coordinates and compares this with data from the Antarctic magnetometers. Halley beam 8 points poleward, along the magnetic meridian, and Halley beam 3 is directed at an angle of $\sim 16^{\circ}$ to the west of the magnetic meridian.

Figure 6a presents the spectral width variation from beam 3 for this interval; the bold line represents the estimated location of the SWB using the same method as discussed in Sect. 2. The poleward distortion of the boundary is very 


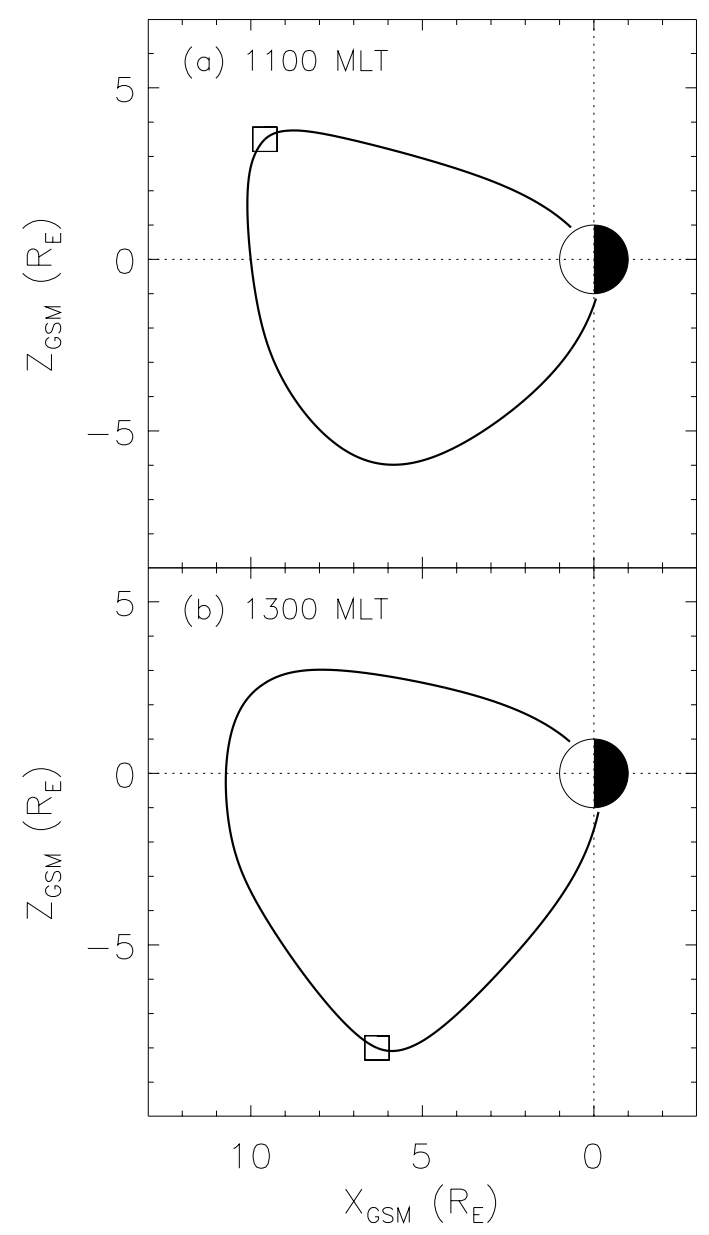

Fig. 7. Model representation of the last closed field line during this interval, in GSM x-z coordinates at (a) 11:00 MLT, and (b) 13:00 MLT. The square symbols represent the locations of the estimated anti-parallel reconnection X-lines.

clear, centred on 11:00 MLT. At later magnetic local times, the boundary is located close to $-67^{\circ}$ latitude, although there appear to be occasional poleward excursions in the boundary. The largest of these excursions (at $\sim$ 15:00 MLT) appears to occur simultaneously on all beams (not shown), suggesting that they may represent temporal, and not spatial variations in the boundary position. Figure $6 \mathrm{~b}$ displays line-of-sight velocity data from Halley beam 3; positive (negative) velocities represent the line-of-sight flow toward (away from) the radar. Two flow regimes are apparent in the line-of-sight velocity data: strong flows above $-71^{\circ}$ latitude in the morning sector and strong flows above $-67^{\circ}$ latitude in the afternoon sector. These flow regimes switch at $\sim 11: 40$ MLT (marked by the vertical dashed line in Fig. 6). There is a clear distinction between the flows and the boundary position observed in the local morning and those observed in the local afternoon. Figure $6 \mathrm{c}$ presents the spectral width variation from beam 8 for this interval. The observed SWB is very similar to that seen by beam 3 in Fig. 6a, albeit at a lower resolution. Figure $6 \mathrm{~d}$ displays the line-of-sight velocity data from beam 8 .
It is immediately obvious that there is not the large distinction between the flow regimes on either side of noon, as seen by beam 3; indeed, the line-of-sight velocities observed by beam 8 suggest that the poleward component of the convection flow is approximately constant on either side of noon. The large afternoon sector flows observed by beam 3 are, therefore, a result of a large increase in the zonal (westward) component of the flow in the afternoon sector.

In order to determine how these flows relate to the largerscale convection pattern, we have studied data from the A80 $\left(-66.89^{\circ}, 27.99^{\circ}\right.$ AACGM coordinates) and A81 (-69.17 ${ }^{\circ}$, $35.81^{\circ}$ AACGM coordinates) AGO magnetometers, located within the Halley radar field of view in the Antarctic. The magnetometer data can provide a crude qualitative estimate of the equivalent convection flow in the dayside ionosphere. The equivalent convection flow is estimated by: First, removing a close, quiet day variation (17 August 1998) from the magnetometer data; second, rotating the residual horizontal magnetic field vector by $90^{\circ}$ anti-clockwise. However, the $H$-component observed by the magnetometers generally provides a good estimate of the east-west component of the equivalent convection, whereas the $\mathrm{D}$ component can be severely affected by large field-aligned currents in the vicinity of the observations (Kamide et al., 1976). Hence, in Fig. 6e, we present the $H$-component variations (with the quiet day variation removed) from A80 and A81, which provide a qualitative estimate of the zonal (east-west) component of the convection flow. Negative values represent eastward flow (as in the dawnside return flow), whereas positive values represent westward flow (as in the duskside return flow). The band around zero represents a region of low zonal flow which corresponds to the throat flow region in the ionospheric convection pattern and provides an estimate of the extent of the merging region. The dotted vertical lines in Fig. 6 represent estimates of the location of the transitions between the return and throat flow regions. The A81 observations do not clearly show a westward flow that represents the duskside return flow. However, it is likely that for much of the interval under study, A81 is located within the polar cap. Although they only provide a crude estimate of the changes in convection, the magnetometer observations suggest that the merging gap (the throat flow region) covers at least $\sim 3 \mathrm{~h}$ of magnetic local time on this day and extends well into the afternoon sector, outside the poleward distortion observed in the morning sector. These afternoon sector flows should correspond to a significant fraction of the observed cross-polar cap potential.

\section{Discussion}

The significant question raised by the observations presented in this paper is why is the SWB in the morning sector (as seen in our localised poleward distortion) located $\sim 2^{\circ}$ poleward of that in the early afternoon sector? This does not represent a temporal change in the PCB position since the distortion is seen as a clearly distinct feature in the spa- 


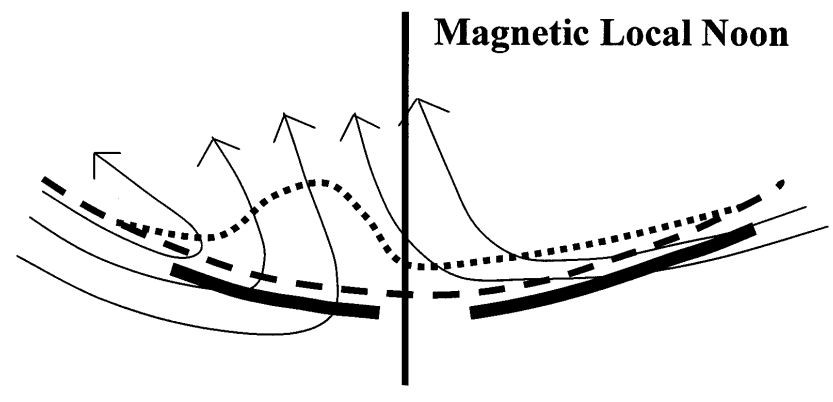

\section{- Polar Cap Boundary ..... Proxy (Spectral Width Boundary) - Extent of Merging Gaps}

Fig. 8. A schematic representation of the relationship between the spectral width boundary, the polar cap boundary, and the convection flow for the interval under study.

tial maps of spectral width (e.g. in Fig. 3). It is also not likely to represent a spatial variation in the actual PCB position as the feature lasts for an extended interval (at least $1 \mathrm{~h}$ ) and any large displacement of the PCB from a stable equilibrium position would be expected to be rectified on a shorter time scale. We can explain the difference as a result of a change in the offset between the SWB and the PCB on either side of magnetic local noon. This could be due either to changes in the flow across the merging gap or to changes in the ion travel time from the reconnection X-line. This becomes an increasingly likely scenario if one considers the consequences of the anti-parallel merging hypothesis (Crooker, 1979). During intervals when IMF $B_{y}$ is comparable to $B_{z}$, the anti-parallel merging hypothesis predicts two distinct reconnection $\mathrm{X}$-lines on the dayside magnetopause (Crooker, 1979; Luhmann et al., 1984; Coleman et al., 2001). For $B_{y} \sim B_{z}<0$, the reconnection X-lines are located at high-latitudes on the Northern Hemisphere magnetopause in the morning sector and on the Southern Hemisphere magnetopause in the afternoon sector.

Figure 7 presents estimates of the morphology of the morning $(\sim 11: 00$ MLT $)$ and afternoon $(\sim 13: 00$ MLT $)$ sectors' last closed field lines for the conditions observed during this event, using the Tsyganenko 96 magnetospheric field model. The squares in Fig. 7 represent the locations on these field lines where the geomagnetic field is antiparallel to a perfectly-draped magnetosheath magnetic field for these conditions (Coleman et al., 2000, for further details of this model). It is immediately obvious from Fig. 7 that the morning sector reconnection X-line is located significantly further from the Southern Hemisphere ionosphere $\left(\sim 21 R_{E}\right.$ fieldaligned distance) than the afternoon sector reconnection $\mathrm{X}$ line $\left(\sim 10 R_{E}\right)$. This implies a longer ion travel time from the reconnection $\mathrm{X}$-line to the ionosphere in the morning sector than in the afternoon sector. If we consider $500 \mathrm{eV}$ protons (the energy at which the cusp ion flux is a maximum, as observed by DMSP-F14), then the difference between the morning and afternoon sector ion travel times is $\sim 230$ s, i.e. significantly longer in the morning sector.

Figure 6d illustrates that the poleward component of the convection flow is similar in both the morning and afternoon sectors. Therefore, the proposed difference in ion travel times would cause the morning sector precipitation to be convected further poleward, explaining the much greater offset between the SWB and the PCB in the morning sector. Assuming a poleward flow component of $\sim 400-500 \mathrm{~m} / \mathrm{s}$ in both the morning and afternoon sectors (Fig. 6d) results in a predicted difference between the morning and afternoon sector SWB offsets of $\sim 92-114 \mathrm{~km}$, i.e. $\sim 1^{\circ}$ latitude. This agrees fairly well with the poleward extent of the distortion observed in the spatial maps of spectral width in Fig. 3. However, Figs. 4 and 6 present variations in the spectral width determined from temporal changes observed by single beams. The UT interval in which the data was collected is likely to have involved a period of polar cap expansion, as expected during an interval of extended southward IMF. Figure 4 illustrates that the SWB dawnward of the edge of the distortion at 10:30 MLT (and dawnward of the merging region, and hence, where we would expect no poleward offset), is located at $\sim-69^{\circ}$. This is at a higher latitude than the SWB observed in the afternoon sector, which may imply an equatorward motion of the PCB over the interval of study.

Another point to note is that the morning sector throat flow appears quasi-steady, whereas the high-resolution data show that the afternoon throat flow is extremely bursty with large line-of-sight velocity flow bursts in evidence. These flow bursts may imply pulsed reconnection and, as a result, transient poleward and equatorward motion of the polar cap boundary (consistent with models such as that of Cowley and Lockwood, 1992). The transient poleward motions of the SWB, evident in the afternoon sector in Fig. 6a, may be a result of this. However, the possibility still exists that much of the SWB motion is a result of transient variations in the offset between the SWB and the PCB and hence, the observed SWB motion may not completely reflect the motion of the PCB.

In Fig. 8, we attempt to summarise the observed scenario in a schematic diagram along the lines of Fig. 1. In the morning sector, the convection flow poleward of the PCB is predominantly poleward (as shown in Figs. 5 and 6). The large ion travel time from the reconnection location on the northern high-latitude magnetopause to the southern ionosphere results in a large offset of the SWB from the PCB, leading to the observed poleward distortion. This distortion images the extent of the morning sector merging region. In the afternoon sector, the convection flow immediately poleward of the PCB is predominantly westward (as shown in Figs. 5 and 6 ), although the poleward component of the flow is similar to that observed in the morning sector (Fig. 6d). The smaller poleward offset of the SWB from the PCB in the afternoon sector is explained as a result of the shorter ion travel time from the reconnection location on the high-latitude Southern Hemisphere magnetopause to the southern ionosphere.

In summary, by studying the characteristics of an unusual 
ionospheric signature of the cusp in the dayside ionosphere during an interval of quasi-steady solar wind conditions, we have been able to study the variations in the factors which determine the offset between the PCB and one of its proxies: the SWB. The lack of variability in the solar wind allowed us to observe a large region of the dayside ionosphere at a time when there was unlikely to be a significant variation in the reconnection scenario on the dayside magnetopause. If one assumes the anti-parallel merging hypothesis, then the difference in the SWB position observed on either side of magnetic local noon arises primarily from the difference between the ion travel time from the morning and afternoon reconnection X-lines on the magnetopause to the Southern Hemisphere ionosphere. These results, therefore, provide evidence to support the anti-parallel merging hypothesis.

This study also highlights that mesoscale or small-scale variations in the SWB do not always represent variations in the PCB. Hence, it is important when using proxies for the PCB to understand the possible variations in the offset from the actual boundary. These variations can result from spatial or temporal changes in the convection flow across the merging gap (i.e. in the reconnection rate), or from variations in the reconnection scenario on the magnetopause, which lead to changes in the ion travel time from the reconnection Xline to the ionosphere. These caveats apply to other proxies for the PCB which are related to cusp particle precipitation, such as the $630 \mathrm{~nm}$ cusp auroral emission. Indeed, it is possible that some of the spatiotemporal structure of the cusp aurora is a result of mesoscale variations of this type.

Acknowledgements. The Halley and Sanae radars were developed under funding from the NERC (UK), NSF (US), and DEAT (South Africa). Operations are supported by the NERC (UK) and DEAT (South Africa). DMSP data and analysis were provided under NSF grant ATM-9713436 and NASA grant NAG5-9297. The WIND data are part of the CDAWeb database. We are grateful to Dr. R. Lepping, principal investigator on the WIND MFI instrument. We would like to thank the Halley SHARE radar engineers, Kevin O'Rourke and David Glynn.

Topical Editor M. Lester thanks K. McWilliams and two other referees for their help in evaluating this paper.

\section{References}

André, R., Pinnock, M., and Rodger, A. S.: On the SuperDARN autocorrelation function observed in the ionospheric cusp, Geophys. Res. Lett., 26, 3353-3356, 1999.

André, R., Pinnock, M., Villain, J.-P., and Hanuise, C.: On the factors conditioning the Doppler spectral width determined from SuperDARN HF radars, Int. J. Geomagn. Aeron., 2, 77-86, 2000.

Baker, K. B., Dudeney, J. R., Greenwald, R. A., Pinnock, M., Newell, P. T., Rodger, A. S., Mattin, N., and Meng, C.-I.: HF radar signatures of the cusp and low-latitude boundary layer, J. Geophys. Res., 100, 7671-7695, 1995.

Baker, K. B., Rodger, A. S., and Lu, G.: HF-radar observations of the rate of magnetic merging: a GEM boundary layer campaign study, J. Geophys. Res., 102, 9603-9617, 1997.
Burke, W. J., Weimer, D. R., and Maynard, N. C.: Geoeffective interplanetary scale sizes derived from regression analysis of polar cap potentials, J. Geophys. Res., 104, 9989-9994, 1999.

Chisham, G., Pinnock, M., and Rodger, A. S.: The response of the HF radar spectral width boundary to a switch in the IMF $B_{y}$ direction: Ionospheric consequences of transient dayside reconnection? J. Geophys. Res., 106, 191-202, 2001.

Coleman, I. J., Pinnock, M., and Rodger, A. S.: The ionospheric footprint of antiparallel merging regions on the dayside magnetopause, Ann. Geophysicae, 18, 511-516, 2000.

Coleman, I. J., Chisham, G., Pinnock, M., and M. P. Freeman: An ionospheric convection signature of antiparallel reconnection, J. Geophys. Res., in press, 2001.

Cowley, S. W. H. and M. Lockwood: Excitation and decay of solar wind driven flows in the magnetosphere-ionosphere system, Ann. Geophysicae, 10, 103-115, 1992.

Crooker, N. U.: Dayside merging and cusp geometry, J. Geophys. Res., 84, 951-959, 1979.

Doyle, M. A., and Burke, W. J.: S3-2 measurements of the polar cap potential, J. Geophys. Res., 88, 9125-9133, 1983.

Greenwald, R. A., Baker, K. B., Dudeney, J. R., Pinnock, M., Jones, T. B., Thomas, E. C., Villain, J.-P., Cerisier, J.-C., Senior, C., Hanuise, C., Hunsucker, R. D., Sofko, G., Koehler, J., Nielsen, E., Pellinen, R., Walker,A. D. M., Sato, N., and Yamagishi, H.: DARN/SuperDARN: A global view of the dynamics of highlatitude convection, Space Sci. Rev., 71, 761-796, 1995.

Hairston, M. R., Heelis, R. A., and Rich, F. J.: Analysis of the ionospheric cross polar cap potential drop using DMSP data during the National Space Weather Program study period, J. Geophys. Res., 103, 26337-26347, 1998.

Heelis, R. A.: The effects of interplanetary magnetic field orientation on dayside high-latitude ionospheric convection, J. Geophys. Res., 89, 2873-2880, 1984.

Kamide, Y., Akasofu, S.-I., and Brekke,A.: Ionospheric currents obtained from the Chatanika radar and ground magnetic perturbations at the auroral latitude, Planet. Space Sci., 24, 193-201, 1976.

Kan, J. R. and Lee, L. C.: Energy coupling function and solar windmagnetosphere dynamo, Geophys. Res. Lett., 6, 577-580, 1979.

Lockwood, M. and Davis, C. J.: On the longitudinal extent of magnetopause reconnection pulses, Ann. Geophysicae, 14, 865-878, 1996.

Lockwood, M.: Relationship of dayside auroral precipitations to the open-closed separatrix and the pattern of convective flow, J. Geophys. Res., 102, 17 475-17487, 1997.

Luhmann, J. G., Walker, R. J., Russell, C. T., Crooker, N. U., Spreiter, J. R., and Stahara,S. S.: Patterns of potential magnetic field merging sites on the dayside magnetopause, J. Geophys. Res., 89, 1739-1742, 1984.

Maynard, N. C., Weber, E. J., Weimer, D. R., Moen, J., Onsager, T., Heelis, R. A., and Egeland, A.: How wide in magnetic local time is the cusp? An event study, J. Geophys. Res., 102, 4765-4776, 1997.

Milan, S. E., Lester, M., Cowley, S. W. H., Moen, J., Sandholt, P. E., and Owen, C. J.: Meridian-scanning photometer, coherent HF radar, and magnetometer observations of the cusp: a case study, Ann. Geophysicae, 17, 159-172, 1999.

Moses, J. J., Siscoe, G. L., Crooker, N. U., and Gorney, D. J.: IMF $B y$ and day-night conductivity effects in the expanding polar cap convection model, J. Geophys. Res., 92, 1193-1198, 1987.

Newell, P. T. and Meng, C.-I.: Ion acceleration at the equatorward edge of the cusp: Low altitude observations of patchy merging, 
J. Geophys. Res., 18, 1829-1832, 1991.

Nishitani, N., Ogawa, T., Pinnock, M., Freeman, M. P., Dudeney, J. R., Villain, J.-P., Baker,K. B., Sato, N., Yamagishi, H., and Matsumoto, H.: J. Geophys. Res., 104, 22 469-22 486, 1999.

Pinnock, M., Rodger, A. S., Baker, K. B., Lu, G., and Hairston, M.: Conjugate observations of the day-side reconnection electric field: A GEM boundary layer campaign, Ann. Geophysicae, 17, 443-454, 1999.

Pinnock, M. and Rodger, A. S.: On determining the noon polar cap boundary from SuperDARN HF radar backscatter characteristics, Ann. Geophysicae, 18, 1523-1530, 2001.

Provan, G., Yeoman, T. K., and Milan, S. E.: CUTLASS Finland radar observations of the ionospheric signatures of flux transfer events and the resulting plasma flows, Ann. Geophysicae, 16, 1411-1422, 1998.

Reiff, P. H., Spiro, R. W., and Hill, T. W.: Dependence of polar cap potential drop on interplanetary parameters, J. Geophys. Res., 86, 7639-7648, 1981.

Reiff, P. H. and Burch, J. L.: IMF $B_{y}$-dependent plasma flow and Birkeland currents in the dayside magnetosphere 2. A global model for northward and southward IMF, J. Geophys. Res., 90, 1595-1609, 1985.

Rodger, A. S., Mende, S. B., Rosenberg, T. J., and Baker, K. B.: Simultaneous optical and HF radar observations of the ionospheric cusp, Geophys. Res. Lett., 22, 2045-2048, 1995.

Rodger, A. S.: Ground-based ionospheric imaging of magnetospheric boundaries, Adv. Space Res., 25, 7/8, 1461-1470, 2000.

Rodger, A. S. and Pinnock, M.: The ionospheric response to flux transfer events: The first few minutes, Ann. Geophysicae, 15, 685-691, 1997.

Ruohoniemi, J. M., Greenwald, R. A., Baker, K. B., and Villain, J.P.: Drift motions of small-scale irregularities in the high-latitude F region: An experimental comparison with plasma drift motions, J. Geophys. Res., 92, 4553-4564, 1987.
Ruohoniemi, J. M. and Greenwald, R. A.: Statistical patterns of high-latitude convection obtained from Goose Bay HF radar observations, J. Geophys. Res., 101, 21 743-21 763, 1996.

Sergeev, V. A., Pellinen, R. J., and Pulkkinen, T. I.: Steady magnetospheric convection: A review of recent results, Space Sci. Rev., 75, 551-604, 1996.

Siscoe, G. L., and Huang, T. S.: Polar cap inflation and deflation, J. Geophys. Res., 90, 543-547, 1985.

Smith, M. F. and Lockwood, M.: Earth's magnetospheric cusps, Rev. Geophys., 34, 233-260, 1996.

Tsunoda, R. T.: High-latitude F-region irregularities: A review and synthesis, Rev. Geophys., 26, 719-760, 1988.

Tsyganenko, N. A.: Modelling the Earth's magnetospheric magnetic field confined within a realistic magnetopause, J. Geophys. Res., 100, 5599-5612, 1995.

Tsyganenko, N. A. and Stern, D. P.: Modelling the global magnetic field of large-scale Birkeland current systems, J. Geophys. Res., 101, 27 187-27 198, 1996.

Villain, J.-P., Caudal, G., and Hanuise, C.: A SAFARI-EISCAT comparison between the velocity of F-region small-scale irregularities and the ion drift, J. Geophys. Res., 90, 8433-8443, 1985.

Weimer, D. R.: An improved model of ionospheric electric potentials including substorm perturbations and application to the Geospace Environment Modeling November 24, 1996, event, J. Geophys. Res., 106, 407-416, 2001.

Wygant, J. R., Torbert, R. B., and Mozer, F. S.: Comparison of S3-2 potential drops with the interplanetary magnetic field and models for magnetopause reconnection, J. Geophys. Res., 88, 57275735, 1983.

Yahnin, A., Malkov, M. V., Sergeev, V. A., Pellinen, R. J., Aulamo, O., Vennerström, S., Friis-Christensen, E., Lassen, K. Danielsen, C., Craven, J. D., Deehr, C., and Frank, L. A.: Features of steady magnetospheric convection, J. Geophys. Res., 99, 4039-4051, 1994. 